\title{
RELAÇÃO DAS OBRAS ENTRADAS NA BIBLIO- TECA DA FACULDADE DURANTE O PERIODO COMPREENDIDO ENTRE 15 DE MAIO E 15 DE AGOSTO DE 1934
}

\author{
OBRAS GERAES-(0)
}

Anais da Assembléa Legislativa Provincial de São Paulo-1837-São Pauło, 1926-1 vol.-Doação.

Anais da Camara dos Deputados do Estado de São Paulo-1912, 1922 e 1924-3 vols.—Doação.

Anais da III ${ }^{a}$ Conferencia Nacional de Educação-São Paulo, 1930-1 vol.Permuta.

Anais do Estado de Mato GrossoCuiabá, 1921-1931-11 viols.--Permuta.

Anais do Estado de Mato GrossoCuiabá, 1931-1 vol.-Permuta.

Anales de La Universidad CentralTomo II-Quito, 1933-1 vol.-Permuta.

Anales de La Universidad de ChileCuarto trimestre de $1933-1$ vol.Permuta.

Anales de La Universidad de Chile1933, 1934-2 vols.-Permuta.

Annals of The American Academy of Political And Social Science. (The-) -Philadelphia, 1934-1 vol.-Compra.

Annaes (The-)-Philadelphia-July, 1934-1 vol.-Compra.

Annuaire de Législation ÉtrangèreParis, 1921, 1926-6 vols.-Doação.

Anuario Forense-Belo Horizonte, 1932 -1 vol.-Permuta.

Arquivos de Assistencia a Psicopatas de Pernambuco-Recife, 1933-1 vol. $\rightarrow$ Permuta.
Arquivo Espirita-N. 1-Publicação pperiodica do Centro Espirita CristãoSão Paulo, 1930-1 vol.-Doação.

Arquivo Judiciario-Publicação do Jornal do Comercio-Rio de Janeiro, 1932-3 vols.-Doação.

Arquivo da Sociedade de Medicina Legal e Criminologia de São PauloVol. IV-1933-S. Paulo, 1934-1 vol. -Permuta.

Archivos de la Universidad de Buenos Aires-Tomo VIII-1933-1 vol.Permuta.

Bibliografia sôbre a organisação internaional do Trabalho-São Paulo, 1933-1 vol.-Doação.

Biblioteca Nacional (La-)-em 1933Buenos Aires, 1934-1 vol.-Permuta.

Boletim da Faculdade Paulista de Letras e filosofia - São Paulo, 1934-1 folh.-Doação.

Boletim do Museu Nacional-V.ol. VIII -Rio de Janeino, 1932-1 vol.-Permuta.

Coletanea de Acórdãos-Bielo Horizonte, 1932-1933-4 vols.-Permuta.

Conde Matarazzo aos oitenta anosMarço, 1934-1 vol.-Doação.

Cencêlho de Contribuintes - 2. ${ }^{\circ}$ volRio de Janeiro, 1934-1 vol.-Compra.

Diario da Assembléa Nacional-Rio de Janeiro, 1934. Encad. 2 vols. Compra.

Diario da Justiça-Rio de Janeiro, 1934 -3 vols.-Compra. 
Duisberg, Carl-Goldenes Berufsjubilaum der Geheimen RegierungsratsLeverkusen, 1933—1 vol.-Doação.

E. Wandenkolk,-Relatorio da viagiem da Corveta Baiana a.o Mar das Indias-Rio de Janeino, 1879-1 vol.Permuta.

Enclyclopaedia of the Social Siciences. -New York, 1934-Vol. XIII-1 vol. _Compra.

“Estado de São Paulo" (O-)-Sĩo Paulo, 1934-2 vols.—Doação.

Estatuto de la Universidad Nacional de Mexico-Mexico, 1934-1 vol.Permuta.

Europe-Revue Mensuelle-Paris, 1934 -1 vol.-Compra.

Feira Literaria--Publicação mensal-São Paulo, 1927-11 vols.-Doação.

Filosofia-Orgão do Centro "D́om Miguel Kruze"-São Paulo, 19311 vol.-Doação.

Finzi (Giuseppe-)-Dizionario di citazioni Latine ed Italiane-Milano1 vol.-Compra.

Grandin, A.-1Bibliographie générale des Sciences Juridiques Politiques, Economiques et Sociales-Paris, 1934 1 vol.-Compra.

Idort-Orgão do Institutio de Organisação Racional do Trabalho-S. Paulo, 1932-1933-2 vols.-Doação.

João Eremita da Silva Ramos-Indice alfabetico das leis e decretos do Es,tado de São Paulo-1889-1926-São Paulo, 1927-1 vol.—Doação.

Jornal do Comercio-Rio de Janeiro, 1934-2 vols.-Compra.

Jurisprudencia Argentina-Buenos Aires, 1918-1933-43 vols. Doação.

Justiça-Porto Alegre, 1934—1 vol.|Permuta.

Larouse Mensuel Illustré-Revue encyclopédique - Paris, 19:29-1931 $\longrightarrow 3$ vols. -Doação.

Meel, J. Van-Bibliotèques Publiques -Traité théorique et pratique-Belgique, 1924-1 vol.-Compra.

Memoria-Balance general y estadistico. Ferrocarriles e Tranvias de Es-
tado.-Ejercicio 1932-33-Montevideo, 1933-1 vol.-Permuta.

Mensaje del Presidente de la Republica Oriental del Uruguay a la Assemblea General-Primer e segundo periodo de Legislatura-Montevideo, $1933-34-2$ vols. - Permuta.

Mois (Le-)-Synthèse de l'activité mondiale-Paris, Abril-Maio, MaioJunho e Junho-Julho, 1934-3 vols. -Compra.

Paraná Judiciario-Vol. XIX-Curitiba, 1934-1 vol.- Permuta.

Opinion Nacional-N. ${ }^{\circ}$ especial-Homenage a la Rep. del Paraguay-B. Aires, Outubro e Novembro de 1933 -1 vol.--Permuta.

Primer Congreso Sanitario Nacional celebrado em Chitre-Panamá, 1934 -1 vol.-Piermuta.

Quinzena Judiciaria (A-) -Ano I-n. ${ }^{\circ}$ 1-Rio de Janeiro, 1927-1 vol.-Doação.

Recueil des Cours-Tables quinquennales-Paris, 1928-1932-1 vol.-Permuta.

Relatorio do ano escolar de 1933-Faculdade de Medicina de Porto Alegre-1 vol.-Permuta.

Relatorio apresentado pela diretoria da Cooperativa dos Empregados da Viação Ferrea do Rio Grande do Sul-Exercicio de 1933-Porto Alegre, 1934-1 vol.-Doação.

Relatorio da Irmandade da Santa Casa de Misericordia de São Paulo. 1933-São Paulo, 1934-1 vol.-Doação.

Relatorio preliminar apresentado á Comissão de Ensino Superior e Universitario de Educação-São Paulo, Janeiro de 1931-1 vol.-Doação.

Rentrée soliennelle des Facultés-Université de Montpellier-Année scolaire 1933-34-1 vol.-Permuta.

Résumé mensual des travaux de la Societe des Nations-Généve, 1929-1930 -2 vols.-Doação.

Revista Argentina de Derecho Internacional-Buenos Aires, 1930-19312 vols.-Doação.

Revista do Arquivo Municipal de São 
Paulo-I e II-São Paulo, 1933/34

2 vols.-Doação.

Revista de Derecho y Ciencias Sociales -Asunción, 1932-33-2 vols.-Permuta.

Revista de Derecho $y$ LegislaciónCaracas-Venezuela, 1933-1 vol.Permuta.

Revista da Faculdade de Direito de São Paulo-Vol. X-São Paulo, 1902 -2 exemplares-Anos de 1893 a 1900 -6 vols.-Compra.

Revista da Faculdade de Filosofia e Letras—São Paulo, 1926-1931—4 vols. -Doação.

Revista do Fôro-Vol. XXVIII-João Pessôa, 1933-1 vol--Permuta.

Revista dos Funcionarios-São Paulo, 1934-1 vol.-Doação.

Revista do Ginásio PernambucanoRecife, 1932 -1 vol.-Doação.

Revista do Instituto de Alagôas-Vol. XVIII-1933-Jaraguá, 1934-1 vol.Permuta.

Revista do Instituto Historico e Greografico da Baía-Ns. 56 e 59-Baía, 1933-1 vol.--PPermuta

Revista do Instituto Historico e Geografico do Pará-Belem, 1922, 1931, 1932, 1933-Vols. 4, 5, 6, 7 e $8-4$ vols. -Permuta.

Revista do Instituto da Ordem dos Advogados da Baia-Baía, 1927-1928 4 vols. - Doação.

Revista de Jurisprudencia BrasileiraVol. XXII-Rio de Janeiro, 1934 1 vol.-Permuta.

Revista Numismatica-Orgão da Sociedarde Numismatica BrasileiraSão Paulo, 1933-1 vol.—Doação.

Revista dos Tribunais-Vol. LXXXVIII -São Paulo, 1933-1 vol.-Permuta.

Revue des Deux Mondes-Paris, 18501890-36 vols.-Compra.

Revue de Droit International PrivéParis, 1930-1931-2 vols.-Doação.

Revue Universelle (La-)-Paris, 19201926-17 vols.-Compra.

Vie Intellectuelle (La-)-Tomo .... XXVIII-Juvisy, 1934-1 vol.-Tomo XXIX-Juvisy, 1934-1 vol.-Permuta.

\section{FILOSOFIA-(1)}

Filosofia, Psicologia, Espiritismo, Moral, etc.

Souza Carneiro, A. J. de-Sciencia Esoterica-São Paulo, 1926-1 vol.-Doação.

Abhedananda, Swani-Filosofia do Trabalho-S. Paulo, 1928-1 vol.- Doação.

Abhedananda, Swani-Como fazer-se Yogi-São Paulo, 1927-1 vol.-Doação.

Alberto Seabra--Versos aureos de Pythagoras-São Paulo, 1928-1 vol.Doação

Alberto Seabra-A alma e o subconsciente-São Paulo, 1927--1 vol.-Doação

Alberto Seabra-O problema do além e do destino- São Paulo, 1927-1 vol. Doação.

Alberto Seabra-Phenomenos Psychicos-S. Paulo, 1927-1 vol.-Doação.

Allem, James-A vida triunfante-São Paulo, 1928-1 vol-Doação.

Allen, James-Da pobreza ao poderSão Paulo, 1933-1 vol.-Doação.

Altavilla, Eurico-Il Suicidio nella psicologia, nella indagine giudiziaria e nel díritto.-Napoli, 1932-1 vol.Compra.

Alvaro Guimarães Filho-Da higiene mental e sua importancia em nosso meio-São Paulo, 1926-1 vol.-Doação.

Antonio Cunha-Da correlação entre testes de desenvolvimento mental e testes psico-motores-São Paulo, 1933 -1 vol.-Doação.

Atkinson, William Walker-O segredo do sucesso-São Paulo, 1931-1 vol. - Doaçãa.

Atkinson, William Walker-A consciencia interna-São Paulo, 19:27-1 vol. -Doação.

Atkinson, William Walker-A lei do novo pensamento-São Paulo, 19281 vol.-Doação.

Atkinson, William Walker-A psicologia do comerciante-São Paulo-1 vol.-Doação.

Atkinson e Beals - 0 Poder creadorSão Paulo, 1931-1 vol.-Doação. 
Atkinson e Beals- $O$ poder ou vosso eu superior-São Paulo, 1931-1 vol. -Doação.

Atkinson e Beals-O poder sub-consciente ou vossas forças secretas-São Paulo, 1931-1 vol.-Doação.

Atkinson e Beals-O poder do desejo -São Paulo, 1931-1 vol.-Doação.

Atkinson e Beals-O poder 'd'a fé-São Paulo, 1931-1 vol.-Doação.

Atkinson e Beals-O poder da vontade —São Paulo, 1931-1 vol.-Doação.

Atkinsen, William Walker-A mente e o corpo ou os estados mentaes e as condições fisicas-São Paulo, 19281 vol.-Doação.

Atkinson, William Walker-A força do pensamento, sua ação na vida e nos negocios-São Paulo, 1929—1 vol.Doação.

Atkinson, William Walker-Leitura pratica do pensamento-São Paulo, 1931 -1 vol.-Doração.

Atkinson e Beals- $O$ poder regenerador ou rejuvenecimento vital-São Paulo, 1931-1 vol.-Doação.

Atkinson e Beals - 0 poder de caracter ou individualidade positiva-São Paulo, 1931-1 vol-Doação.

Atkinson e Beals-O poder do raciocinio ou logica pratica-São IPaulo, 1931-1 vol.-Doação.

Atkinson e Beals-O poder die percepção ou a arte de observação-São IPaulo, 1931-1 vol.-Doação.

Atkinson e Beals - $O$ poder espiritual -São Paulo, 1931-1 vol.-Doação.

Atkinson e Beals- $O$ poder do pensamento ou radio-mentalismo-S. Paulo, 1931-1 vol.-Doação.

Atkinson, William Walker-Fascinação mental-São Paulo, 1928-1 vol.Doação.

Atkinson, William Walker-Magia mental ou segredo do poder mental-São Paulo, 1929-1 vol.-Doação.

Barlet, F. Ch.-O ocultismo-São Paulo, 1916-1 vol.-Doação.

Barlet, F. Ch.-Ensaio sobre a evolução da idéa-São Paulo, 1917-1 vol. - Doação.

Bernheim, H.-Automatisme et suggestion-Paris, 1917-1 vol.-Doação.
Bertho Condê-Ensaio de politica espiritualista-São Paulo, 1927-1 vol. -Doação.

Camaysar, Rosabis-Psycho-analyse pratica-São Paulo, 1931-1 vol.-Doação.

Camaysar, Rosabis-Caminho da realisação-São Paulo, 1931-1 vol.Doação.

Camaysar, Rosabis-Os doze mêses iniciaticos e as nove esferas da mentalidade-São Paulo, 1931-1 vol.Doação.

Camaysar, Rosabis-Fraternidade cosmica-São Paulo, 1932-1 vol.-Doação.

Camaysar, Rosabis-Consciencia cosmica-São Paulo, 1933-1 vol.-Doação.

Catú, Potyra-Força magica-São Paulo, 1930-1 vol.-Doação.

Cicero-A vontadie-obra posthuma de Cicero-São Paulo, 1925-1 vol.-Doação.

Collins (Mabel)-Pelas portas de Ouro -S. Paulo, 1929-1 vol.-Doação.

Curso de Iniciação Esoterica-São Paulo, 1933-1 vol.-Doação.

Devoe, Walter-0 segredo da concentração-São Paulo, 1933-1 vol.-Doação.

Dicionario de Ciências Ocultas-São Paulo, 1931-1 vol.-Doação.

Doutrina Secreta dos RosacrucianosSão Paulo, 1929-1 vol.-Doação.

Dreser, Horacio W.-O livro dos segredos-São Paulo, 1927-1 vol.-Doação.

Dumas, Georges-Nouveau Traité de Psychologie-Tome troisième-Paris, 1933-1 vol.-Compra.

Durville, Heitor-Magnetismo pessoal ou psychico-São Paulo, 193ऋ-1 vol. -Doação.

Durville, Henri-Força atrativa do pensamento-São Paulo, 1929-1 vol.Doação.

Durville, Henri-Misterios inciaticosSão Paulo, 1926-1 vol.-Doação.

Encausse, G.-A reencarnação-S. Paulo, 1927-1 vol.-Doação.

Eurico de Goes-A corrente filosofica do seculo-São Paulo, 1926-1 vol.Doação.

Evangelho de Rômakrishna-São Paulo, 1925-1 vol.-Doação. 
Ferriére-La Doctrine de Spinoza 1 vol.-Compra.

Finot, Jean-Prolonguemos a vidaSão Paulo, 1931-1 vol.-Doação.

Forças Ocultas-São Paulo, 1934-1 vol. -Doação.

Forel, Augusto-Etica sessuale-Torino -1 vol.-Doação.

Fouillée, Alfred-La Psychologie des Idées-Forces-Paris, 1893-2 vols.Comprá.

Francisco Ribeiro dos Santos e Ennio Monteiro Galembeck-Psicologia e logica-São Paulo, 1934-1 vol.-Doação.

Frank, Henry-Esoterismo do casamento-São Paulo, 1933-1 vol.-Doação.

Gêtâ, Bhagavad-A sublime canção tda imortalidade - São Paulo, 1924—1 vol. -Doação.

Hara, O. H.Psichometrica praticaSão Paulo, 1932-1 vol.-Doação.

Hara, O. H.-Alchimia mental ou prodigios da força do pensamento-São Paulo, 1926-1 vol.-Doação.

Harnold, Hans-O adepto ou ensinamentos da alta magia-São Paulo, 1930-1 vol.-Doação.

Hilaire, J. Barthelem y-Politique D'Aristote-Paris, 1837-2 vols-Compra.

Homem e seus corpos ( $0_{-}-$- Os sete elementos ocultos do homem-São Paulo, 1932-1 vol.-Doação.

João Francisco-Escola Republicana estoica-Rio de Janeiro, 1934-2 vols. -Doação.

Kaibalion, O-Estudo da filosofia Hermetica do antigo Egypto e GreciaSão Paulo, 1924-1 vol.-Doação.

Karl, Miguel-O espiritualismo na India e a filosofia Vedanta-São Paulo, 1928-1 vol.-Doação.

Larson, Christian D.-A maravilhosa fonte interna-Os prodigios do inconsciente-São (Paulo, 1928-1 vol.Doação.

Levi, Eliphas-Grande Arcano ou o ocultismo desvendado-S. Paulo, 1926 -1 vol.-Doação.

Livro do exito ou força do pensamento -São Paulo, 1931-1 vol.-Doação.

Loester-Poder magico-S. Paulo, 1930 -1 vol-Doação.

Loester-Praticas esotericas-São Paulo, 1930—1 vol.-Doação.
Luz no Caminho-São Paulo, 1929-1 vol.-Doação.

Mallard, G.-Les Merveilles de L'Autor suggestion-Paris, 1924-1 vol.-Daação.

Mange, Roberto-Psychotecnica-S. Paulo, 1934-1 vol.-Doação.

Mann, G. A-O homem completo e sua atitude mental-São Paulo, 1931-1 vol.-Doação.

Mann, G. A.-A aniciativa, a coragem e a audacia-São Paulo, 1932-1 vol. -Doação.

Maria Lacerda de Moura-Religiáo do amôr e da beleza-São Paulo, 1929 1 vol.-Droação.

Max-Palavras do Infinito-São Paulo, 1923-1 vol.-Doação.

Método de hipnotismo-São Paulo, 1928 -1 vol.-Doação.

Mulfor, Prentice-Nossas forças mentaes- São Paulo, 1933 — 4 vols.-Doação.

Naillem, A. Van Der-A grande mensagem-São Paulo, 1928-1 vol.-Doação.

Naillem, A. Van Der-No SantuarioSão Paulo, 1933—1 vol.-Doação.

Naillem, A. Van Der-Nos Templos do Himalaya- São (Plaulo, 1933-1 vol. -Doação.

Naillem, A. Van Der-Bailthazar o Mago

-São Paulo, 1933-1 vol.-Doação.

Noções Elementares de Kabbala-São Paulo-1 vol.-Doação.

Ottolenghi, Salvatore-La suggestione e le facoltá psichiche occulte-Torino, 1900-1 vol.-Permuta.

L'Ospedale Psichiatrico de S. Niccolo in Siena della- Società di Esecutori di Pie Disposizioni-(1818-1933)-Siena, 1933-1 vol.-Doação.

Papus-Que é o ocultismo-São Paulo, 1928-1 vol.-Doação.

Papus-Traité méthodique de sciences occultes-Parris, 1891-1 vol.-Daação.

Prasad, Yogi Rama-As forças subtis da natureza-São Paulo, 1926-1 vol. -Doação.

Prasad, Yogi Rama-Ciência da respiração-São Paulo, 1926-1 vol.-Doação. 
Ramacharaka, Yogi-Raja Yoga ou desenvolvimento mental-S. Paulo, 1928 -1 vol.-Doação.

Ramacharaka, Yogi-A vida depois da morte-São Paulo, 1926-1 vol.-Doação.

Ramacharaka, Yogi-A ciência IndúYogi da respiração-São Paulo, 1934 -1 vol.-Doação.

Ramacharaka, Yogi-Gnani Yoga ou a Yoga da sabedoria-São Paulo, 1926 -1 vol.-Doação.

Ramacharaka, Yogi-Cristianismo místico-São Paulo, 1926-1 vol.-Doação.

Ramacharaka, Yogi--Hatha Yogi do bem-estar fisico-São Paulo, 1932-1 vol.-Doação.

Ramacharaka, Yogi-As doutrinas esotericas das filosofias e religiões da India-São Paùlo, 1931-1 vol.-Doação.

Ramacharaka, Yogi-Quatorze lições de filosofia Yogi-São Paulo, 1932-1 vol.-Doação.

Ramacharaka, Yogi-Cura pratica pela agua-São (Plaulo, 1930-1 vol.-Doação.

Rassegna di Studi Psichiatrici-Indici generali dell'anno 1911-vol. I-all'anno 1932--vol. XXI-Siena, 1934 1 vol.-Permuta.

Régis, N. Pitres y E.-Las obsesiones y los impulsos-Madrid, 1910-1 vol.Doação.

Tagore, Rabindranath-Gitan jali-São Paulo, 1929-1 vol.-Doação.

Tarde, G-Les lois de l'ImitationParis, 1890-1 vol.-Compra.

O Templo do Silencio-São Paulo, 1931 -1 vol.-Doação.

Trine, Ralph Waldo-Regras para o viajor—São Paulo, 1924-1 vol.-Doação.

Trine, Ralph Waldo-A lei da vidaSão Paulo, 1933-1 vol.-Doação.

Trine, R. W.-Harmonia com o Infinito-São Paulo, 1926-1 vol.-Doação.

Turnbull, V.-Curso de magnetismo pessoal ou arte de triumphar na vida -São Plaulo, 1933-1 vol.--Doação.

Vivekananda, Swami-Filosofia Vedanta-São Paulo, 1932-1 vol.-Doação.

Vivekananda, Swami-Rajor Yoga e outros assuntos-São Paulo, 1931-1 vol.-Doação.
Walker, Edward-Os pensamentos são cousas-São Paulo, 1931-1 vol.-Doação.

Weston, Walter Newell-A intuiçãoSão Paulo, 1931-1 vol-Doação.

\section{RELIGIÕES-(2)}

A. J. de Souza Carneiro-Jesus !...São Paulo, 1927-1 vol.-Doação.

D'Alveydre, Saint-Yves-Mission des Juifs-Paris, 1884-1 vol.—Compra.

Francisco H. Rodrigues Valle-Sympsychotheismo-São Paulo, 1919-1 vol. -Doação.

Martins, J. P. Oliveira-Systema dos Mythos Religiosos-Lisboa, 1895-1 vol.-Compra.

Taboado, Luiz Brono-El Patrono de los Congressos Eucaristicos San Pascual Bailon-Buenos Aires, 1934-1 vol.-Plermuta.

Vivekananda, Swami-Estudos da religião-São Paulo, 1931—1 vol-Doação.

CIENCLAS JURIDICAS E SOCLAIS, SOCIOLOGIA, POLITICA, ESTATISTICA, ECONOMIA, DIREITO, MEDICINA

LEGAL, EDUCAÇÃO, COSTUMES, ETC.-(3)-

A. F. Cesarino Junior-Sociedades anônimas estrangeiras-São Paulo, 1934 -1 vol.-Doação.

A. Romano Barreto-Organização das Penitenciarias - com rapildo estudo sobre o crime e o criminoso-São Paulo, 1934-1 vol.-Doação.

Abelardo Marinho-O sufragio profissional-Rio de Janeiro, 1934-1 vol. -Doação.

Acondlãos do Tribunal da Relação do Estado de Minas Gerais-1924-1929Belo Horizonte, 1928-1933-7 vol.Permuta.

Afranio Peixoto - Sexologia forenseRio de Janeiro, 1934-1 vol.-Compra.

Alberto Pessoa-Guia de técnica policial-Coimbra, 1929-1 vol.-Compra.

Alcantara Machado-Medicina Publica -Preleções-1 vol.-Permuta

Alcortã, Amancio-Curso de Derecho Internacional Privado--Buenos Aires, $1927-3$ vols.-Permuta. 
Almachio Diniz-O Poder revolucionario e a liberdade de profissão-Rio de Janeiro, 1933-1 vol-Doação.

Almachio Diniz-O que é preciso reformar: o criterio nacional do nosso povo-Rio de Janeiro, 1933-1 vol.Doação.

Almachio Diniz-Os tratados Argentino-Brasileiros-Rio de Janeiro, 1933 -1 vol.-Doação.

Almachio Diniz-O imperialismo norteamericano-Rio de Janeiro, 1933-1 vol.-Doação.

Almachio Diniz-Erros do falso racionalismo constitucional de Mirkine Guetzvitch e de Vicente Ráo-Rio de Janeiro, 1933-1 vol.-Doação.

Almachio Diniz-O monstro- (o antigo projecto da Constituição Federal)Rio de Janeiro, 1933-1 vol.-Doação.

Almachio Diniz-Confederacionismo imperioso-Rio de Janeiro, 1933-1 vol. -Doação.

Almachio Diniz-Duvidas sobre um testemunho historico da revoluçãoRio de Janeiro, 1933-1 vol.—Doação.

Antonio Luiz da Camara Leal-Manual elementar do Direito Civil-São Paulo, 1930-3 vols-Compra.

Antonio Pinto Cardozo de Mello-Desquite-São Paulo, 1934—1 vol.-Doação.

Arbassier, Charles-L'Absolutisme en Bourgogne-L'Intendant Bouchu et son action financière-Dijon, 19191 vol.-Permuta.

Armando Valente Junior-Da responsabilidade moral e legal dos medicos -São Paulo, 1929-1 vol.-Doação.

Arnaldo Codespoti-Contribuição ao estudo das unhas em medicina legal -São Paulo-1 vol.-Doação.

Arthur de Carvalho Moreira-Da execução dos julgamentos estrangeiros em materia civil-São Plaulo, 19341 vol.-Doação.

Ascarelli, A-Compendio di Midicina Legale-Roma, 1912-1 vol.-Permuta.

Aspectos da Questão Monetaria-Artigos de um observador financeiro, a proposito do relatorio do Snr. Otto Niemeyer-São Paulo, 1931-1 vol.Doação.
Aspectos sociales de la racionalizacion -Estudios preliminares (Los)-Madrid, 1932-1 vol.-Compra.

Assistence Publique de la Ville de Strasbourg-Budget de L'exercicie, 1934-1 vol.-Permuta.

Azael Simões Luitner-Pesquiza Toxico-logica do bismuto e sua dosagem colorimetrica-São Paulo, 1933-1 vol. -Doação.

Bendicente, Francisco C.-El Metodo en la investigacion y exposicion de las materias economicas-Rosario, 1933-1 vol.-Doação.

Bijon, S.-Étude Critique de L'Évolution de L'Impot de Succession-Paris, 1927-1 vol.-Compra.

Brasilio Pereira de Souza-Contribuição para o estudo da determinação da Idade das fraturas-São Paulo, 1933 -1 vol.-Doação.

Bruno Rudolfer e W. Leser-Estatistica-São Paulo, 1934-1 vol.-Doação.

Bryce-Les Démocraties Modernes-Paris, 1924-1 vol._Compra.

Butera, Antonio-Delle TransazioniTorino, 1933-1 vol. Compra.

Caio Nunes de Carvalho-Nova democracia: nova Republica-Rio de Janeiro, 1931-1 vol.-Doação.

Carlos A. Turano-Considerações sobre as lesões corporaes.-S. Paulo, 1931 -1 vol.-Doação.

Carlos Costa-Chrystaes de hemoglobina em medicina legal-São Paulo, 1933-1 vol.-Doação.

Carlos Maximiliano-Hermenêutica e aplicação do direito-Porto Alegre, 1933-1 vol.-Doação.

Carlos Pasquale-Sobre duas modificações do metodlo de Strzyzonvski, para obtenção dos cristaes de TeichmannSão Paulo, 1932-1 vol.-Doação.

Carqueja, Bento-Economia P'oliticaPorto-4 vols.-Compra.

Carreira, Liberato de Castro-Historia Financeira e orçamentaria do Imperio do Brasil-Rio de Janeiro, 1889 -1 vol.--Compra.

Carvalho Borges, E. M. de-O principio de Direito, de acôrdo com a concepcão teleologica-S. Paulo, 19341 vol.-Doação. 
Casaurance, J. M. Puig-La autonomia de la Universidiad-Discurso-Mexico, 1934-1 vol.-Doação.

Catalano, E.-La riforma penale e i suoi riflessi educative-Palermo, 1930 -1 vol.-Doação.

Cicero de Almeida Morais-Verificação da reação de Lecha-Marzo (para manchas de esperma)-S. Paulo, 1929-voll.-Doação.

Clausing, Auguste-Essai critique sur les conceptions du Droit Allemand en matière de publicité legale des sociétés de commerce-Paris, 19291 vol.-Doação.

Clovis Bevilaqua-Codigo Civil dos Estados Unidos do Brasil-comentado -III vol.-Rio de Janeiro, 1933-1 vol.-Permuta.

Codigo Civil Brasileiro InterpretadoV.ol. V-Rio de Janeiro, 1934-1 vol. CCompra.

Codigo Municipal de Obras-S. Paulo, 1933-1 vol.-Compra.

Codigo Penal (Ley numero 9155)—Montevidéo, 1930-1 vol.-Permuta.

Coleção das Leis e Decretos do Estado de São Paulo-Tomo XLIIY-S. Paulo, 1933-1 vol.-Doação.

Comision General de Reclamaciones entre Panamá y Estados Unidos de America-Panamá, 1934-1 vol.-Permuta.

Communisme en Chine (Le-)-19321 vol.-Doação.

Constituição da Republica dos Estados Unidos do Brasil-S. Paulo, 1934-1 val.-Compra.

Convencion celebrada entre la Republica de Panamá y los Estados Unidos de America para construcion de um canal-Panamá, 1924 -1 vol.Permuta.

Cooperativismo y Socialismo-Montevideo, 1921-1 vol.-Permuta.

Cours de 1933-Académie de Droit International de La Haye—Paris, 1933 -1 vol.-Doação.

Couture, Eduardo J.-El divarcio por voluntad de la mujer-Montevideo, 1931-1 vol.- Plermuta

Conture, Eduardo J.-Teoria de las diligencias para mejor prover-Montevideo, $1932-1$ vol.-Permuta.
Couture, Eduardo J.-La acción declarativa de prescripcion-Montevideo, 1933-1 vol.-Doação.

Cristovam Mangione-Contribuição para o estudo da identificação pela marcha-São Paulo, 1931-1 vol.-Doação.

Curt Egon Reichert-A adoção-São Paulo, 1934-1 vol.-Doação.

Davis, Horace B.-Economia SocialSão Paulo, 1934-1 vol.-Doação.

Dechesne, Laurent-Économie industrielle et sociale-Paris, 1932-1 vol. - Permuta.

Decio Ferraz Alvim-Concepção institucional do direito-São Paulo, 1934 -1 vol.-Dotação.

Decio Ferraz Alvim-Uma nova concepção do direito e o corporativismo -Rio de Janeiro, 1934-1 vol.-Doação.

Decretos e Atos do Municipio de S. Paulo-São Paulo, 1932-1933-2 vols. -Doação.

Delgado, Juan C. Quinteros-Proteccionismo industrial-Montevideo, 19181 vol.-Plermuta.

Diogo José da Silva Netto-Filosofia do Direito-A concepção do direito na nova sociedade-São Paulo, 1934 -1 vol.-Doação.

Djacir Menezes-Introdução á ciencia do direito-Porto Alegre, 1934-1 vol. $\neg$ Doação

Djalma Forjás-Ensaios de um quadro demonstrativo do desmembramento das comarcas do Estado de São Paulo-S. Paulo, 1931-1 vol.-Permuta.

Djalma Forjás-Ensaios de um quadro demonstrativo do desmembramento dos municipios do Estado de São Paulo-São Paulo, 1931-1 vol.-Permota.

Doctrines monetaires à l'epreuve des faits, (Les-) Conférences-Paris, 1931-1 vol. - Permuta.

Documentos elucidativos-Inquerito do Instituto de Café do Estado de São IPaulo-N.s. 1 e 2-São Paulo, 19332 vols.-Doação.

Domingos de Oliveira Ribeiro NetoProtecção dos interesses moraes e materiais do medico-São Paulo, 1928 -1 vol.-Doação. 
Duguit, Léon-Manuel die Droit Constitutionnel-Paris, 1907-1 vol.-Compra.

E. M. de Carvalho Borges-Como devem ser preenchidas as lacunas da legislação comercial brasileira-São Paulo, 1934-1 vol.-Doação.

Edison de Oliveira-Considerações medico-legaes sobre os ferimentos leves e graves-São Paulo, 1932-1 vol. $\rightarrow$ Doação.

Edmundo Auernig Burle-Direito Internacional Privado-São Paulo, 1934 -1 vol.-Doação.

Ehrt, Adolf-Révolte armée-BerlinLeipzig, 1933-1 vol:-Permuta.

El plan sexenal de Mexico-Rio de Janeiro, 1934-1 vol.-Doação.

Ernestino Lopes da Silva Junior-Determinação da idlade pelo estudo dos dentes-S. Paulo, 1931-1 vol.-Doação.

Estudio de las cuestiones planteadas a la Comision de Conciliación con la Municipalidade de Buenos Aires -Octubre, 1933-Enero 1934-1 vol. -Permuta.

Fausto D'Oliveira Quaglia-Da experimentação do testemunho em psicologia legal-São Paulo, 1929-1 vol. -Doação.

Financial and Economic Annual of Japan-1 vol.-Doação.

Flora, Federico-Manuale della Scienza delle Finanze-Livorno, 1921-1 vol. -Compra.

Fouillée, Alfred-L'Idée moderne du droit-Paris-1 vol.-Doação.

Frederico Ferrigno-O método crioscópico médico-legal da morte por intoxicação alcoolica-São Paulo, 1934 -1 vol.-Doação.

G. de Almeida Moura-O Nacional-socialismo alemão-São Paulo, 1934-1 vol.-Doação.

Garay, Narciso-Tradiciones y cantares de Panamá-1930-1 vol.-Permuta.

Goldberg, Anna-Essai d'une theorie générale de l'engagement juridique par volonté unilatérale d'aprés le Code Civil Allemand-Paris, 1913-1 vol.-Permuta.
H. Almeida Gomes-Racionalisação de discriminação e da arrecadação de rendas-Conferencia-Rio de Janeiro, 1934-1 vol.-Doação.

Heiden, Konrad-Histoire du National Socialisme-1919-1934-Paris, 1934-1 vol-Compra.

Henry Couannier, André-Elementos creadores del Derecho Aéreo-Madrid, 1929-1 vol.-Compra.

Hermenegildo Urbina Telles-O Medico e a politica-São Paulo, 1929. vol.-Doação.

Hermes Lima-Introdução á ciência do Direito-São ıPaulo, 1934-1 vol.Compra.

Inquerito do Instituto 'de Café-Documentos elucidativos-S. Paulo, 1934 -1 vol.-Doação.

Iolando Mirra-Contribuição para o estudo medico-legal da crioscopia do sangue-S. Paulo, 1931-1 vol.-Doação.

J. M. de Carvalho Santos-Codigo civil brasileiro interpretado-Vol. IVRio de Janeiro, 1934-1 vol.-Compra.

J. de Oliveira Filho-Do conceito da ordem publica-São Paulo, 1934-1 vol.-Doação.

J. P. Calogeras-A politica exterior do Imperio-Rio de Janeiro, 19272 vols.-Permuta.

J. X. Carvalho de Mendonca-Pareceres-Rio de Janeiro, 1934-1 vol.Compra.

João Baptista de Oliveira e Costa Janior-Contribuição para o estudo das "linhas brancas dactyloscopicas" e d.o seu valor na identificação-São Paulo, 1933 - 1 vol.-Doação.

João Ferraz do Amaral-Escolas ao ar livre-São Paulo, 1932-1 vol.-Doação.

João Junqueira Franco-Do emprego do sôro precipitante anti-humano seco na reação de Uhlenhuth-São Paulo, 1933-1 vol.-Doação.

João Pimenta-Quadro de Titulos da Bolsa de São Paulo-1934-1 vol.Doação.

João Teixeira da Silva Braga-A especialisação em medicina-São Pauło, 1930-1 vol.-Doação. 
João Vicente de Luca-Do valor dos elementos cytologicas na pericia das manchas-São Paulo, 1927-1 vol.Doaşão.

Joaquim Ferreira da Rocha-A deformidade nas lesões pessoaes-S. Paulo, 1931-1 vol.-Doação.

Jorge Americano-Codigo do Processo Civil e Comercial do Estado de São Paulo-1.` Viol.-São Paulo, 1934-1 vol.-Doação.

Jorge Severino-Curso de critica penal Rio de Janeiro, 1934-1 vol.-Doação.

José Augusto Lefévre- $\mathrm{Da}$ hereditariedade dos grupos sanguineos e sua aplicação na investigação da paternidade-S. Paulo, 1928-1 vol.-Doação.

José Carlos de Macedo Soares-Autonomia Municipal-Discurso-São Paulo, 1934-1 vol.-Doação.

José Dias da Silveira-Estudios praticos para a identificação dos alcaloides pelo metodo microquimico coin o reativo de Dragendorff-São Paulo, 1933-1 vol.-Doação.

José Mauricio Corrêa-A puberdade feminina em São Praulo em suas relações medico-legaes-S.ão Paulo, 1931 -1 vol.-Doação.

José Prudente de Aquino-Contribuição para o estudo de revisão dos cristais de hemocromogenio-São Paulo, 1934-1 vol.-Doação.

Julgados-Tribunal Superior de Ala. gôas-1911-1918-Maceió, 19:22-19232 vols.-Permuta.

Jurisprudencia-Diario da Justiça-Vol. 7. - 1 vol.-Compra.

Kantorowicz, Hermann U.-La lotta per la scienza del diritto-Milano, 1908-1 vol-Doação.

Lemos Britto-A questão sexual nas prisões—Rio de Janeiro, 1934-1 vol. -Doação.

Ley 11 de 1932 sobre sueldos y asignaciones, $y$ sobre fijacion del personal administrativo de las oficinas de la Republica-Panamá, $1932-1$ vol.Permuta.

Ley 26 de 1932 sobre imigracion- $\mathrm{Pa}$ namá, 1933-1 vol.-Permuta.

Leyes 41 de 1925 y 26 de 1928 sobre servicio Diplomatico y ConsularPanamá, 1929-1 vol.-Permuta.
Leyes de Registro Civico Nacional de Ciudadania legal $y$ de EleccionesMontevideo, 1931-1 vol.-Permuta.

Linneu Prestes-Da analise e eliminação da cocaina e da morfina pela saliva, nos casos clinicos de cocainomania e die morfinomania-S. Paulo -1 vol.-Doação.

Lombroso, Gina-La tragedia del progreso-Madrid, 1932-1 vol.-Compra.

Lonvrie, Samuel-Sociologia geral-São Paulo, 1933-1934-1 vol.-Doação.

Luiz Pinto de Toledo-Contribuição para o estudo das chamadas "Psicoses de prisão"-São Paulo, 1934-1 vol.-Doaçãio.

Magalhães, Barbosa de La doctrine du domicile en Droit International Privé-Paris, 1929-1 vol.-Doação.

Magalhães, Barbosa de-Plaidoiries du Délégué du gouvernement de la République Portugaise-Lisboa, 1928-1 vol.-Doação.

Magalhães, Barbosa de-Mémoire justificatif des réclamations portugaises-Lisboa, 1933-1 vol.-Doação.

Magalhães, Barbosa de-La nationalité des sociétés de commerce d'apres la législation portugaise-Famalicão, 1932-1 vol-Doação.

Magalhães, Barbosa de-“Laws of Maritime Jurisdiction in time of peace" and "Maritime Neutrality"-Conference-Lisboa, 1927-1 vol.-Doação.

Magalhães, Barbosa de-A obra do espanhol Fray Francisco de VitóriaA fundação dos direitos das gentes e 0 atual direito internacional- $\mathrm{Fa}$ malicão, 1928-1 vol.-Doação.

Magalhães, Barbosa de $\longrightarrow$ Seguro contra acidentes de trabalho.-Lisboa, 1913 -1 vol.-Doação.

Manuel Viotti-Vicios de conformação das mãos-São Paulo, 1934-1 vol.Doação.

Mariana Coelho-Evolução do feminismo-Rio de Janeiro, 1933-1 vol.Compra.

Mario Brasil Cocaci-A consolidação das fraturas e a lei dos acidentes do trabalho-São Paulio, 1927-1 vol.Doação.

Mello Nogueira, J. F. de-A propaganda do Brasil no Exterior-São Paulo, 1912-1 vol.-Doação. 
Mello Nogueira, J. F. de-Acção de investigação da paternidade-Apelação civel n. $9.990-S$. Paulo, 1919-1 vol. -Doação.

Mello Nogueira, J. F. de-Circulez Messieurs-Habeas Corpus requerido em favor da população paulista, vitima dos desmandos da policia-1 vol.Doação.

Miguel Paulo Capalbo-Sociedade anônima estrangeira-São Paulo, 19341 vol.- Doação.

Milton Olyntho de Arruda-Da identificação do recem-nascido-São Paulo, 1926-1 vol.-Doação.

Mirabelli, Giuseppe-Della Prescrizione -Napoli, Torino, 1915-1 vol--Compra.

Morató, Octavio-El mecanismo de la vida económica-Montevideo, 19331 vol.-Doação.

Nicolau Nazo-A proteção das minorias nas sociedades anônimas-TeseSão Paulo, 1934 -1 vol.-Doação.

Normandin, André-Du Sitatut Juridique des Assocjations Internationales -Paris, 1926-1 vol.-Permuta.

Odecio Bueno de Camargo-Patologia do juri-São Paulo, 1934-1 vol.Doação.

Oliveira, 'Artur Aguedo de- $\mathrm{O}$ imposto de rendimentos na teoria e na pratica-Coimbra, 1923-1 vol-Compra.

Olivi, Luigi-Diritto Internazionale Pubblico-Milano, 1933—1 vol.-Compra.

Oscar de Andrade Coelho-Dos efeitos extraterritoriais da sentença declaratoria da falencia-São Paulo, 1934. 1 vol.l.-Dotação.

Oscar Moura Abreu-Contribuição para o estudo da dosagem da uréa no sangue "ante" e "post-mortem" em suas aplicações medico-legais-São Paulo, 1931-1 vol.-Doação.

Oscar Ribeiro de Godoy-Contribuição para o estudo da identificação das penas-S. Paulo, 1934-1 vol.-Doação.

Oswaldo Aranha Bandeira de MelloA Teoria das Constituições Rigidas -São Paulo, 1934-1 vol.-Doação.

Oswaldo Ferraz Alvim-Sucessão dos estrangeiros no Brasil-São Paulo. 1934-1 vol.-Doação.
Otto Gil-Novo regulamento das vendas mercantis-Rio de Janeiro, 1932 -1 vol. - Permuta.

Otto Gil-As leis de familia da Russia Sovietica perante a justiça brasileira -Rio de Janeiro, 1932-1 vol.-Doação.

Otto Gil-As leis de flamilia da Russia Sovietica perante a justiça brasileira -Rio de Janeiro, 1932-2.a edição1 vol.-Doação.

Otto Gil-Em torno do art. $8 .^{\circ}$ do Codigo Civil-Rio de Janeiro, 1928-1 vol.-Doação.

Paulo Americo Passalacqua-Magistratura Paulista-Discurso-1934-1 vol. -Doação.

Paulo C. de Azevedo Antunes-Eugenia e imigração-São Paulo, 1926-1 vol. -Doação.

Paulo C. Costa-Homicidio casual-S. Paulo, 1934-1 vol.-Doação.

Pedro José de Carvalho-Dos Reclamos Medicos-S. Paulo, 1933-1 vol. -Doção.

Pelloux, Robert-Le Problime du Domaine Public-Paris, 1932-1 vol.Compra.

Philip, André-Le Probléme ouvrier aux Etats-Unis-Paris, 1927-1 vol.Permuta.

Philippe Aché Junior-Responsabilidade criminal do embriagado-São Paulo, 19.33-1 vol.-Doação.

Antonio Piccarolo-ll Fenomeno Frola -São Paulo, 1934-1 vol.-Doação.

Plinio de Lima-Grupos sanguineos em cadaveres-Da imutabilidade dos grapos sanguineos "Pos't mnrtem"-São Paulo, 1932-1 vol.-Doação.

Ponton, Luiz Sanchez-Las Deudas Exteriores-Mexico, 1934-1 vol.-Doação.

Posada, Adolfo-El Régimen Municipal de la ciudad moderna-Madrid, 1916 -1 vol.-Compra.

Pouvourville, A. de-Criffes rouges sur L'Asie-Paris-1 vol.-Doação.

Prus, A.-Ciéncia Penal e Direito Positivo-Lisboa, 1915-1 vol-Compra.

Puglia, Ferdinando-L'Evoluzione Storica e Scientifica del Diritto e della Procedura Penale-Messina, 1882-1 rol.-Permuta. 
Quesada, Ernesto-La evolucion del Derecho Publico-Buenos Aires, 19341 vol.-Permuta.

Reajustamento economico dispondo sobre a redução dos debitos de agricultores-Rio de Janeiro, 1934-1 vol.Doação.

Recopilacion de leyes correspondente al ministerio de gobierno-Buenos Aires, 1925-2 vols.-Permuta.

Registro de usos comerciais. Cheques visados-Rio de Janeiro, 1930-1 vol. -Doação.

Renato Marcondes de Lacerda- 0 instituto da Hipoteca-São Paulo, 1934 -1 vol.-Doação.

Renato Paes de Barros-Da formação da vontade diretora do Estado-São Paulo, 1934-1 vol.-Doação.

René Mendes de Oliveira-Contribüção para o estado da Mancha Negra da Esclerotica-S. Paulo, 1933-1 vol. -Doação.

Revista da Propriedade Industrial-Rio de Janeiro, 1934-3 vols.-Compra.

Ripert, Lucienne-La Reparation du Préjudice dans la Responsabilité Délictuelle-Paris, 1933-1 vol-_Compra.

Roberto Gomes Caldas Filho-Contribuição para o estudo medico-legal da's manchas pellos raios ultra violeta-S. Paulo, 1932-1 vol.-Doação.

Rocco, Alfredo-Principios de Direito Comercial-São Paulo, 1931-1 vol.Compra.

Rodolfo Rocha Ribeiro-Filosofia do Direito-Do direito como proteção do individuo, na sociedade-São Paulo, 1934-1 vol. - Doação.

Ruy de Azevedo Sodré-Função Social da Propriedade Privada-São Paulo, 1934-1 vol.-Doração.

Ruy de Castro Quintanilha-Da identificação do recem-nascido-São Paulo-1 vol.-Doação.

Salvador Pinto Junior e Otto Gil-Copropriedade contratual mista da sociedade-S. (Plaulo, 1933-1 vol.-Doação.

Schoenberg, Gustavo-Trattato di Scienze delle Finanzi-Torino, 1915-1 vol. -Compra.
Schoulguine, Vassili-La Résurrection de la Russie-Paris, 1927-1 vol.Doação.

Scott, James Brown-Die richterliche Entscheidung von internationalen Streitfallen-Breslau, 1933-1 vol.Doação.

Scott, Jamen Brown-The Spanish Origin of International Law-London, 1934-1 vol-Permuta.

Sofovich, Manuel-La Tragedia Boliviana-Buenos Aires, 1932-1 vol.-Permuta.

Sylla Orlandini Mattos-O contagio venereo sifilitico-São Paulo, 1929 1 vol.-Doação.

Synesio Rocha-O crime de envenenamento em face da nossa legislação social-S. Paulo, 1934-1 vol.-Doação.

Tratado celebrado entre la Republica de Panamá y los Estados Unidos de America-1 vol-Permuta.

Vecchio, Giorgio Del-Le Probléme des Sources du Droit Positif-Paris, 1934 -1 vol.--Doação.

Vecchio, Giorgio Del-Giustizia e Diritto-Roma, 1934-1 vol.-Doação.

Velarde, Fabian-Analisis del nuevo tratado-Panamá, 1927-1 vol.-Permuta.

Verger, Henri-Evolucion del concepto medico sobre la responsabilidad de los delincuentes-Madrid, 1922-1 vol. -Compra.

Verginaud, Ernest-Enrichissement sans cause-Paris, 1916-1 vol.-Compra.

Vicente de Paulo Vicente de Azevedo -Crime, Dano-Reparação-São Paulo, 1934-1 vol.-Doação.

Viriato Fernandes Nunes-As perversões sexuaes em medicina legal-São Paulo, 1928-1 vol.-Doação.

Walter Sidney Pereira Leser-Contribuição para o estudo dos metordos estatisticos aplicaveis á medicina e a Higiene-São Paulo, 1933-1 vol.Doação.

Zappa, Gino--Le Valutazioni di Bilancio-Milano, 1927-1 vol.-Compra.

Zeballos, E. S.-La Nationalité-Paris, 1916-1919-2 vols.-Permuta.

\section{FLLLOGIA E LINGUISTICA-(4)}

Lorenz, F. V.—Iniciação linguisticaSão Paulo, 1929-1 vol.-Doação. 
Novo Manual da Lingua PortuguezaRio de Janeiro-1 vol.-Doação.

Vecchi, Emilio Augusto-Gramatica da Lingua Italiana-Lisboa, 1901-1 vol. -Doação.

\section{CIENCLAS PURAS-(5)}

Cabrera, Angel-Zoogeografia-Ciclo de conferencias, $\longrightarrow$ Buenos Aires, 1933-1 vol.-Permuta.

Domingos Jaguaribe- 0 Imperio dos Incas no Perú e no Mexico-S. Paulo, 1927-1 vol.-Doação.

Ekblom, R.-Die Lettischen Akzentarten-Uppsala, 1933-1 vol.-Doação.

Estellita Ribas-Exame prenupcial-S. Paulo, 1927-1 vol.-Doação.

Fiquier, Louis-L'Homme primitif-Paris, 1882-1 vol.-Compra.

Girod, Paul-Les Sociétes chez les Animaux-Paris, 1891-1 vol.-Compra.

Guarany Sampaio-A esterilização eugenica e a deontología medica-São Piaulo, 1928-1 vol.-Doação.

Haberlandt, Michael-Etnografia-Buenos Aires, 1929-1 vol.-Compra.

Huxley, Th. H.-La Place de L'Homme dans la nature-Paris, 1891-1 vol.Compra.

Lanessan, J. L.-La Lutte pour L'Existence ret L'Evolution des SociétésParis, 1903-1 vol.-Compra.

Mello Moraes Filho-Os ciganos no Brasil-Rio de Janeiro, 1886-1 vol. -Doação.

Nordau, Max-Dégénérescence-Paris, 1894-2 vols.-Compra.

Paulo de Godoy-Eugenia e seleção-S. Paulo, 1927-1 vol.-Doação.

Pedro Monteleone - Os cinco problemas da eugenia brasileira-S. Paulo, 1929 -1 vol.-Doação.

Rodrigues de Meréje- 0 problema da Raça-São Paulo, 1934-1 vol.-Doação.

\section{CIENCIAS APLICADAS-(6)}

Abel Augusto de Moura-Cardiopatias dos escolares-São Paulo, 1931-1 vol. -Doação.

Arne Ragnar Enge-Do controle medico da educação fisica-São Paulo, 1929-1 vol.-Doação.
Clovis Corrêa-Inspeção preliminar da malaria-São Paulo, 1926-1 vol.Doação.

Eduardo Monteiro-Propedeutica respiratoria-São Paulo, 1925-1 vol.Doação.

Eduardo Monteiro-Clinica Medica-S. Paulo, 1924-1 vol.-Doação.

Jayme Candelaria-Questões de assistencia-São Paulo, 1931-1 vol.-Doação.

Jenny, Ernest G.-Las fraudes en contabilidad-Barcelona-Madrid-1 vol.Compra.

João Ferraz Monteiro-Contribuição ao estudo radiologico das mastoiditesEstudo radiologico do osso temporal -São Paulo, 1934-1 vol.-Doação.

João de Moraes Junior-Contribuição para o estudo sanitario do sorvete na cidade de São Paulo-São Paulo, 1933-1 vol.-Doação.

José Fernandes de Almeida-O diabete em face da medicina preventiva-S. Paulo, 1932-1 vol.-Doação.

José Lentino-Problema da sifilis congenita e sua profilaxia-São Paulo, 1928-1 vol.-Doação.

Lucinda Romano-Pesquiza e dosagem do Iodo na materia organica-São Paulo, 1933-1 vol.-Doação.

Luiz Maragliano Junior-Da sifilis congenita e sua profilaxia-São Paulo, 1928-1 vol.—Dołação.

Mario de Souza Cotrim-Do abastecimento higienico do leite-São Paulo, 1931-1 vol.-Doação.

Nelson Silveira Corrêa-Problema higienico das piscinas em São PauloSão Paulo, 1932-1 vol.-Doação.

Nelson de Souza Campos-Da tuberculose infantil e sua profilaxia-Vacinação preventiva pelo B. C. G.-São Paulo, 1927-1 vol.-Doação

Paulo Villela de Andrade-Serviço de verificação de obitos em São Paulo -São Paulo, 1933-1 vol.-Doação.

Rubens Azzi Leal-o leite, seu controle higienico pela prova redutase-São Paulo, 1934-1 vol.-Doação.

Ulysses Paranhos-Manual de Therapeutica clinica-São Paulo, 1927-1 vol-Doação. 
Waldemar Beifort Mattos-As Sarcophagas de São Paulo-S. Paulo, 1926 -1 vol.-Doação.

\section{BELAS ARTES-(7)}

Tourist Guide-Panamá-1 vol.-Permuta.

\section{LITERATURA-(8)}

Adriano de Mendoza-Memorias de um'Alma-São IPaulo, 1928-1 vol.Doação.

Amaury Fonseca-Maria das Dôres-S. Paulo, 1926-1 vol.-Doação.

Berilo Neres-A costela de Adão-contos-Rio de Janeiro, 1929-1 vol.Doação.

Borgoño, Luiz Barros-Através de uma correspondencia-Dan Juan Maria Gutiérrez-Santiago, $1934-1$ vol.Permuta.

Carlos Maul-Barbaros-Poemas da terra brasileira-Rio de Janeiro, 1927 -Doação.

Carlos Sampaio-Conferencias-Ribeirão Preto, 1923-1 vol-Doação.

Donoso,' Ricardo-Antonio José die Irisarri escritor y diplomatico-Santiago, 1934-1 vol.-Permuta.

Gil Vicente-Obras de Gil VicenteI, II, III vols.-Lisboa, 1852-3 vols. - Permuta.

Goethe, W.-Faust et le second Faust -Paris-1 vol.-Compra.

J. M. de Azevedo Marques e Ernesto Leme-Discursos-São Paulo, 19341 vol.-Doação.

João Pandia Calogeras-Res nostra... São Paulo, 1930-1 vol.-Piermuta.

José Carlos de Macedo Soares-Discurso pronunciado na Assembléa Nacional Constituinte--São Paulo, 19341 vol..-Doação.

Laforque, Jules-Oeuvres complètesParis, 1922 -5 vols.-Permuta.

Lytton, Eduardo Bulwer-Zanoni-Romance oculista-São Paulo, 1934 1 vol.-Doação.

Lytton, Eduardo Bulwer-O filho de Zanoni-Romance oculista-S. Paulo, 1931-1 vol.-Doação.

Martin Francisco-Contribuindo-São Paulo, 1921-1 vol.-Doação.
Mello Nogueira-0 Ermitão que se fez Diabo-Contos-São Paulo, 1928 -1 vol.-Doação.

Mello Nogueira-O Espelho magicoSão Paulo, 1927-1 vol.-Doação.

Mello Nogueira-Pela mão das mulheres-Contos-São Paulo, 1926-1 vol. -Doação.

Mistral, Frédéric-Oeuvres de Frédéric Mistral-Mireille-Paris-1 vol.Compra.

Monteiro Lobato-Idéas de Jeca Tatú -São Paulo, 1920-1 vol.-Doação.

Osorio Dutra-Dentro da noite azulRio de Janeiro, 1934-1 vol.-Doação.

Paes Barreto Filho-Brasilidade - Discurso-Manáos, 1925-1 vol.-Doação.

Rogerio Gordilho de Faria-Missão cultural do Juiz moderno-Discurso -Baía, 1934 -1 vol.-Dotação.

Suriñach, José-Zoraida-Romance-S. Paulo, 1924-1 vol.-Doação.

Suriñach, José-Gioconda-São Paulo, 1931-1 vol.-Doação.

Suriñach, José-Alarico de AntegueraSão Paulo, 1925-1 vol.-Doação.

Ulisses Paranhos-Idéas e comentarios -São Paulo, 1926-1 vol.-Doação.

Veiga Miranda-Faiscadores, 1925-1 vol.-Doação.

Voragine, Jacques de-La Légende Dorée-lParis, 1902-1 vol.-Compra.

HISTORIA, GEOGRAFIA POLITICA, VIAGENS, BIOGRAFIA, HERALDI-

$$
\text { CA, etc.-(9) }
$$

Affonso José de Carvalho-O novo Bispo de São Clarlos-ConferenciaSão Paulo, 1934-1 vol-Doação.

Armando D'Aguiar-Oliveira SalazarO homem e o ditador-São Paulo, 1934-1 vol.-Compra.

Béraud, Henri-Ce que j'ai vu à Moscou-Paris-1 vol.-Doação.

Chavez, Ezequiel A.-El primero de los grandes educadores de la AmericaFray Pedro de Gante-Mexico, 19341 vol.-Permuta.

Clovis Ribeiro-Brazões e Bandeiras do Brasil-São Paulo, 1933-1 vol.Doação.

Fernando Gallage-A revolução dos Farrapos-São Paulo, 1934-1 vol:Doação. 
João Baptista Martins de MenezesNotas biograficas como funcionario publico e magistrado-São Paulo, 1934 - 1 vol.-Doação.

Iorga, N.-Essai de synthése de L'Histoire de L'humanité-Paris, 19264 vols.-Permuta.

Ladisláo dos Santos Titára-Memoria do grande Exercito Aliado Libertador do Sul da America-Rio Grande do Sul, 1852-1 vol.-Permuta.

La Pradelle, A. de $\longrightarrow$ L'Amerique à Montevideo-Paris, 1934 1 vol.-Permuta.

Libro de Cabildos de Quito-Quito, 1934-4 vols.-Doação.

Lincoln-Porto Alegre, 1934-1 vol.Doação.
Magalhães, Barbosa de-Elogio historico de D. Antonio Mendes Melo-Discurso-Lisboa, 1931-1 vol.-Doação.

Mello Nogueira, J. F. de—Excursão a Mato-Grosso-S. Paulo-1 vul.-Doação.

Nicolau Dreys-Noticia descritiva da Provincia do Rio-Grande de São Pedro do Sul-Rio Grande, 1927-1 vol. -Doação.

Salvador de Mendonça-Apontamentos biograficos para historia das campanhas do Uruguay e Paraguay desde 1864-Rio de Janeiro, 1866-1 vol.Permuta.

Spencer, Herbert-Une Autobiographie -Paris, 1907-1 vol.-Compra. 